Pacific

Journal of

Mathematics

\title{
NUMBER OF SINGULARITIES OF \\ STABLE MAPS ON SURFACES
}

TAKAHIRO YAMAMOTO 


\title{
NUMBER OF SINGULARITIES OF STABLE MAPS ON SURFACES
}

\author{
TAKAHIRO YAMAMOTO
}

\begin{abstract}
Let $N$ denote the plane $\mathbb{R}^{2}$ or the 2-sphere $S^{2}$. In this paper, we determine the 5-tuples of integers $(g, d, i, c, n)$ such that there exists a degree $d$ stable map $\Sigma_{g} \rightarrow N$ whose singular point set consists of $i$ connected components, $c$ cusps, and $n$ nodes, where $\Sigma_{g}$ is the standard genus $g$ surface.
\end{abstract}

\section{Introduction}

Throughout this paper, all surfaces and manifolds are connected and of class $C^{\infty}$ (i.e., smooth), and all maps are of class $C^{\infty}$. Let $M$ be a closed surface and $N$ be a surface. For a $C^{\infty}$ map $\varphi: M \rightarrow N$, denote by $S(\varphi)$ the set of singular points of $\varphi$. Call $\varphi(S(\varphi))$ the apparent contour (contour for short), and denote it by $\gamma(\varphi)$. In this paper, all $C^{\infty}$ maps $M \rightarrow N$ have nonempty singular point sets.

A $C^{\infty}$ map $\varphi: M \rightarrow N$ is said to be stable if it satisfies the following two properties.

(1) For each $p \in M$, the map germ of $\varphi$ at $p$ is $C^{\infty}$ right-left equivalent to one of the map germs at $0 \in \mathbb{R}^{2}$ as follows:

$$
(a, x) \mapsto \begin{cases}(a, x), & p \text { is a regular point }, \\ \left(a, x^{2}\right), & p \text { is a fold point, } \\ \left(a, x^{3}+a x\right), & p \text { is a cusp point. }\end{cases}
$$

Hence, $S(\varphi)$ is a disjoint union of finitely many circles.

(2) For each $q \in \gamma(\varphi)$, the map germ $\left(\left.\varphi\right|_{S(\varphi)}, \varphi^{-1}(q) \cap S(\varphi)\right)$ is right-left equivalent to one of the three multigerms as depicted in Figure 1.

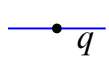

Fold

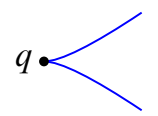

Cusp

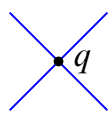

Node

Figure 1. The multigerms of $\left.\varphi\right|_{S(\varphi)}$.

MSC2010: primary 57R45; secondary 57M20, 58K15.

Keywords: stable map, cusp, node. 
According to a classical result of Whitney [1955], stable maps form an open dense subset of the space of all $C^{\infty}$ maps $M \rightarrow N$ with respect to the Whitney $C^{\infty}$ topology.

For a stable map $\varphi: M \rightarrow N$, denote by $c(\varphi), n(\varphi)$ and $i(\varphi)$ the numbers of cusps, nodes and connected components of $S(\varphi)$, respectively.

For a nonnegative integer $g$, the closed and oriented surface of genus $g$, which is the connected sum of $g$ copies of the 2-dimensional torus $T^{2}$, is denoted by $\Sigma_{g}$. The 2-dimensional sphere and the plane are denoted by $S^{2}$ and $\mathbb{R}^{2}$, respectively.

For any stable map $f: M \rightarrow S^{2}$ (or $\mathbb{R}^{2}$ ) of a closed and oriented surface $M$, one can associate the 5-tuple of integers $(g, d(f), i(f), c(f), n(f))$, where $g$ is the genus of $M$ and $d(f)$ is the mapping degree of $f$. This paper studies the following question: which 5-tuples $(g, d, i, c, n)$ can occur in this way?

Some necessary conditions have been obtained by Pignoni [1993], Kamenosono and Yamamoto [2009] (see also Proposition 3.4), Eliashberg [1970], and Quine [1978] (see also Theorem 3.11 of the present paper). M. Yamamoto [2009] studied the numbers $i(f)$ of fold maps $f: \Sigma_{g} \rightarrow \Sigma_{h}$.

András Szúcs posed the following question at the International Workshop on Real and Complex Singularities, held in São Carlos in 2012: whether these conditions form a complete set of restrictions.

The answer is No. There is a geometrical condition for the number of nodes. More precisely, there is the minimal number of nodes for a given 4-tuple $(g, d, i, c)$. The main results of this paper are the following two theorems.

Let $\mathrm{v}_{1}=(2,1)$ and $\mathrm{v}_{2}=(0,2)$ be vectors in $\mathbb{R}^{2}$. For given integers $k, \ell \geq 0$, denote by $L_{k, \ell}$ the affine lattice $\left\{\mu_{1} \mathrm{v}_{1}+\mu_{2} \mathrm{v}_{2}+(2,0) \mid \mu_{1}, \mu_{2} \in \mathbb{Z}\right\}$ if $k \equiv \ell \bmod 2$, and the lattice $\left\{\mu_{1} v_{1}+\mu_{2} v_{2} \mid \mu_{1}, \mu_{2} \in \mathbb{Z}\right\}$ otherwise. For given integers $k, \ell \geq 0$, set $\delta_{k, \ell}=2$ if $k \equiv \ell \bmod 2$, and $\delta_{k, \ell}=0$ otherwise.

Theorem 1.1. Let $g \geq 0$ and $i \geq 1$. If $f: \Sigma_{g} \rightarrow \mathbb{R}^{2}$ is a stable map whose singular point set consists of $i$ components, then the pair $(c(f), n(f))$ is in $L_{i, g} \cap D$, where $D$ denotes the subset of $\mathbb{R}^{2}$ (expressed by coordinates $(x, y)$ ) defined by the following:

$$
D= \begin{cases}\left\{(x, y) \mid x \geq \delta_{i, g}, y \geq 0, y \geq-\frac{1}{2} x+g-i+3, y \geq \frac{1}{2} x-g-i+1\right\} \\ \left\{(x, y) \mid x \geq \delta_{i, g}, y \geq 0, y \geq \frac{1}{2} x-g-i+1\right\} & \text { if } 1 \leq i \leq g\end{cases}
$$

Furthermore, for any pair $(c, n)$ in $L_{i, g} \cap D$, there is a stable map $f: \Sigma_{g} \rightarrow \mathbb{R}^{2}$ with $S(f)$ consisting of $i$ components, $c$ cusps, and $n$ nodes.

Theorem 1.2. Let $g, d \geq 0$ and $i \geq 1$. If $f: \Sigma_{g} \rightarrow S^{2}$ is a degree $d$ stable map whose singular point set consists of $i$ components, then the pair $(c(f), n(f))$ is in $L_{i, g+d} \cap D$, where $D$ denotes the subset of $\mathbb{R}^{2}$ (expressed by coordinates $(x, y)$ ) defined by the following: 
$g=0:$

$$
D= \begin{cases}\{(x, y) \mid x \geq 2(d+1-i), y \geq 0\} & \text { if } 1 \leq i \leq d \\ \left\{(x, y) \mid x \geq \delta_{i, d}, y \geq 0\right\} & \text { if } i \geq d\end{cases}
$$

$g \geq 1:$

$$
\begin{aligned}
& \left\{\begin{array}{r}
(1) \quad\left\{(x, y) \mid x \geq \delta_{i, g}, y \geq 0, y \geq-\frac{1}{2} x+g+3-i\right\} \\
\text { if } d=0 \text { and } 1 \leq i \leq g,
\end{array}\right. \\
& \text { (2) }\left\{(x, y) \mid x \geq \delta_{i, g}, y \geq 0\right\} \quad \text { if } d=0 \text { and } i>g \text {, } \\
& \text { (3) }\left\{(x, y) \mid x \geq 2(d+1-g-i), y \geq 0, y \geq-\frac{1}{2} x+d+g+3-i\right\} \\
& \cup\{(x, y) \mid x \geq 2(d+g+1-i), y \geq 0\} \\
& \text { if } d \geq 1 \text { and } 1 \leq i \leq d-g+1 \text {, } \\
& \text { (4) }\left\{(x, y) \mid x \geq \delta_{i, g+d}, y \geq 0, y \geq-\frac{1}{2} x+d+g+3-i\right\} \\
& \cup\{(x, y) \mid x \geq 2(d+g+1-i), y \geq 0\} \\
& \text { if } d \geq 1 \text { and } d-g+1 \leq i \leq d+g-1 \text {, } \\
& \text { (5) }\left\{(x, y) \mid x \geq \delta_{i, g+d}, y \geq 0\right\} \quad \text { if } d \geq 1 \text { and } i \geq d+g \text {. }
\end{aligned}
$$

Furthermore, for any pair $(c, n)$ in $L_{i, g+d} \cap D$, there is a degree $d$ stable map $f: \Sigma_{g} \rightarrow S^{2}$ with $S(f)$ consisting of $i$ components, $c$ cusps and n nodes.

In Theorems 1.1 and 1.2, generators $\mathrm{v}_{1}$ and $\mathrm{v}_{2}$ correspond to two modifications for stable maps between surfaces: $\mathrm{v}_{1}$ is passing through the swallow-tail singularity (Figure 2), while $\mathrm{v}_{2}$ is passing through the tangency singularity (Figure 3).

In order to prove Theorems 1.1 and 1.2, we will construct maps for any 5-tuples in the list. The constructions go as follows. There are ten modifications that can apply to any map in order to obtain a map with a new 5-tuple.

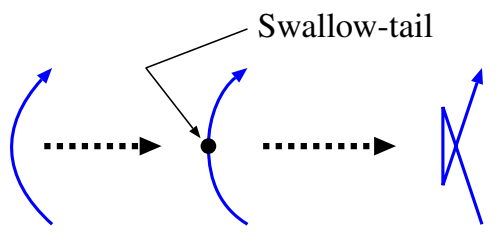

Figure 2. Swallow-tail singularity.

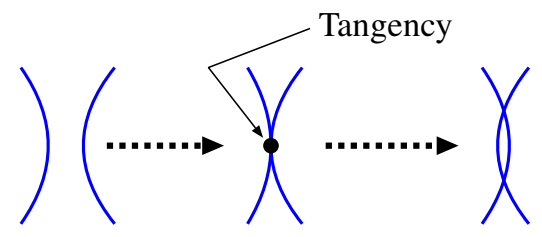

Figure 3. Tangency singularity. 
(1) Passing through the swallow-tail singularity (Figure 2):

$$
(g, d, i, c, n) \rightarrow(g, d, i, c+2, n+1),
$$

(2) Passing through the tangency singularity of the singular curve (Figure 3):

$$
(g, d, i, c, n) \rightarrow(g, d, i, c, n+2),
$$

(3) Attaching two spheres (Figure 4):

$$
(g, d, i, c, n) \rightarrow(g, d, i, c+4, n),
$$

(4) Attaching a handle vertically (Figure 5, left):

$$
(g, d, i, c, n) \rightarrow(g+1, d, i+1, c, n),
$$

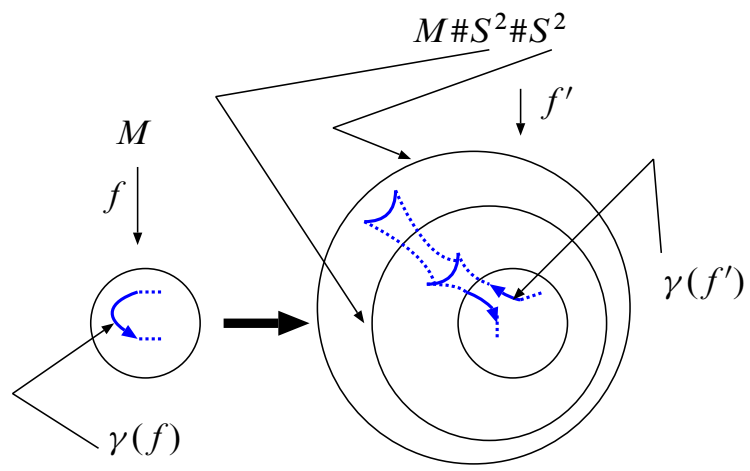

Figure 4. Attaching two spheres: by attaching two maps $\mathrm{id}_{S^{2}}$ and $-\operatorname{id}_{S^{2}}$ to $f: M \rightarrow S^{2}$, we obtain a stable map $M \rightarrow S^{2}$, where $\operatorname{id}_{S^{2}}$ denotes the identity map on $S^{2}$ and $-\mathrm{id}_{S^{2}}$ the $C^{\infty}$ map of $S^{2}$ into $S^{2}$ defined by $x \mapsto-x$.
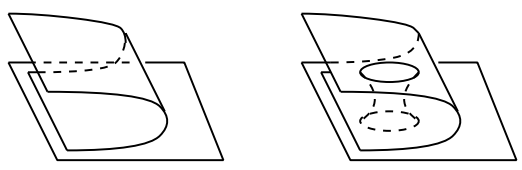

Attach a handle
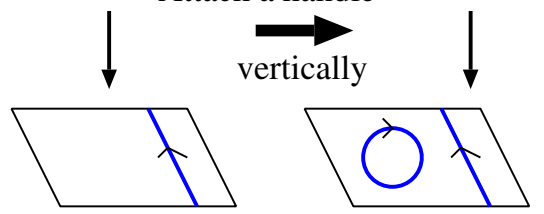
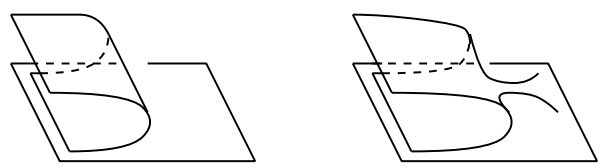

Attach a handle

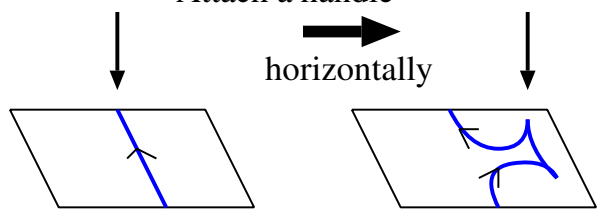

Figure 5. Left: attaching a handle vertically. Right: attaching a handle horizontally. The map is obtained when one projects these surfaces to the horizontal plane. 


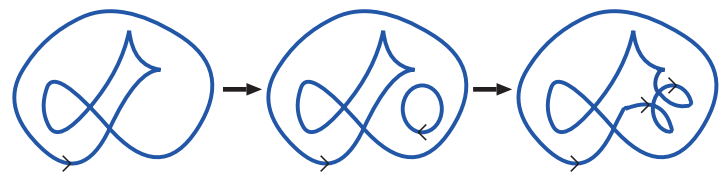

Figure 6. Attaching a pair of handles: attaching a handle vertically and then attaching a handle horizontally.
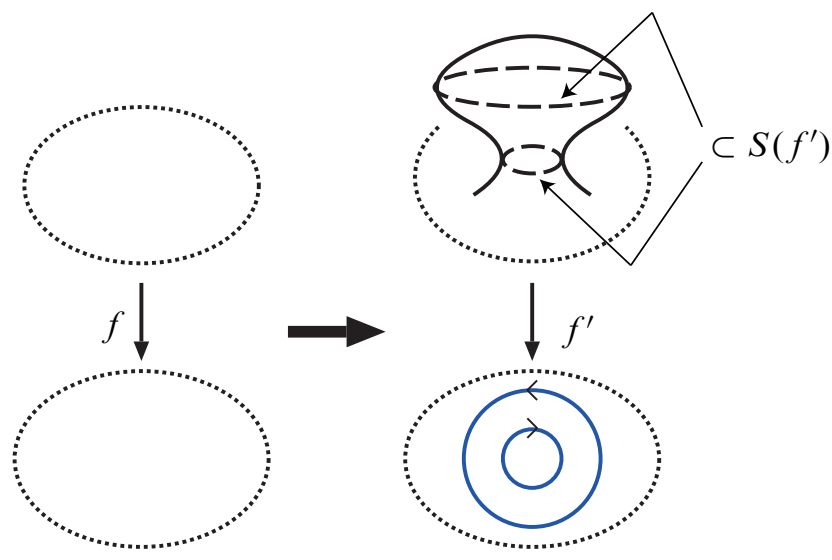

Figure 7. Attaching a balloon.

(5) Attaching a handle horizontally (Figure 5, right):

$$
(g, d, i, c, n) \rightarrow(g+1, d, i, c+2, n),
$$

(6) Attaching a pair of handles. More precisely, attaching a handle vertically and then attaching a handle horizontally (Figure 6):

$$
(g, d, i, c, n) \rightarrow(g+2, d, i, c, n+2),
$$

(7) Attaching a balloon (Figure 7):

$$
(g, d, i, c, n) \rightarrow(g, d, i+2, c, n),
$$

(8) Making a wrinkle (Figure 8):

$$
(g, d, i, c, n) \rightarrow(g, d, i+1, c+2, n),
$$

(9) Attaching a sphere horizontally (Figure 9):

$$
(g, d, i, c, n) \rightarrow(g, d+1, i, c+2, n),
$$

(10) Attaching a sphere vertically (Figure 10):

$$
(g, d, i, c, n) \rightarrow(g, d+1, i+1, c, n) .
$$



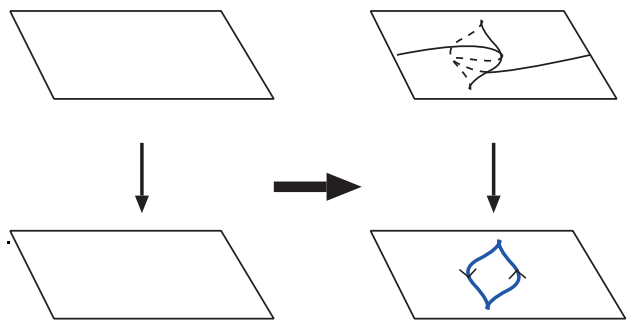

Figure 8. Making a wrinkle.
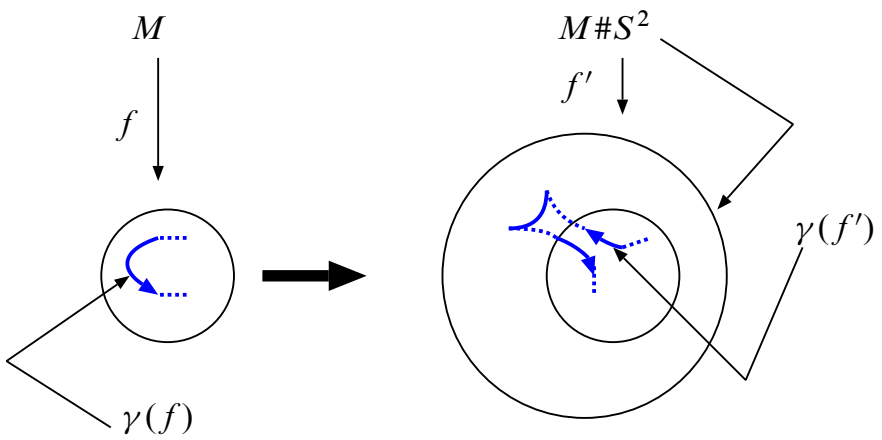

Figure 9. Attaching a sphere horizontally.
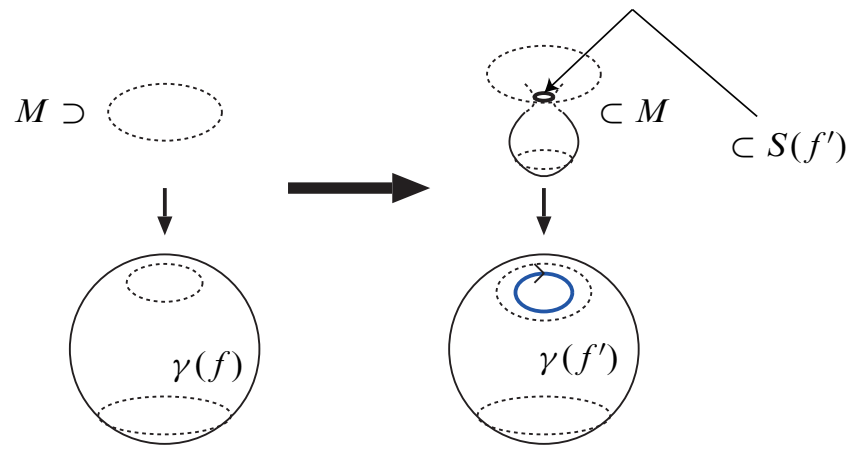

Figure 10. Attaching a sphere vertically.

(11) Attaching a pair of a sphere and a handle. More precisely, attaching a sphere vertically and then, attaching a handle horizontally (Figure 11):

$$
(g, d, i, c, n) \rightarrow(g+1, d+1, i, c, n+2),
$$

We remark that we can apply all modifications $(i)$ with $1 \leq i \leq 11$ to stable maps of surfaces into the sphere. But we can apply modifications (1) and (2), (4), (5), (6), (7), (8) to stable maps of surfaces into the plane. 


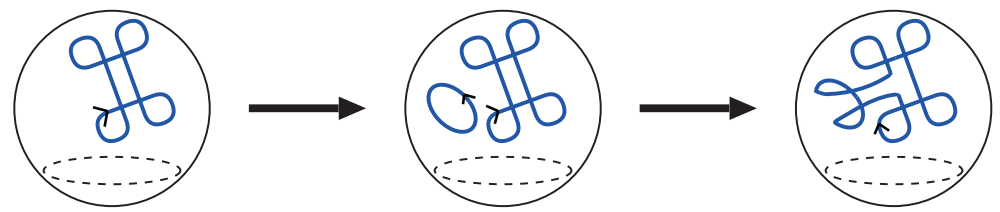

Figure 11. Attaching a pair of a sphere and a handle: attaching a sphere vertically and then, attaching a handle horizontally.

These modifications never decrease the number of 5-tuple $(g, d, i, c, n)$, but they increase some of them. Hence it is enough to construct maps providing the minimal 5-tuples. These constructions can be found in [Demoto 2005; Fukuda and Yamamoto 2011; Kamenosono and Yamamoto 2009; Yamamoto 2009; Yamamoto 2010]. We will sketch their descriptions in Section 2.

Remark 1.3. Theorems 1.1 and 1.2 together with the previous results [Fukuda and Yamamoto 2011; Kamenosono and Yamamoto 2009; Pignoni 1993; Yamamoto 2009; 2010] make the very first step toward classifying generic $C^{\infty}$ maps of closed surfaces into the plane or the sphere up to right-left equivalence.

Remark 1.4. Let $M$ be a closed surface and $N$ a surface. Let $\mathbb{A}$ be an element, an ordered pair, or triple consisting of some elements in $\{c, i, n, c+n\}$. For a stable map $\varphi: M \rightarrow N$, denote by $\mathbb{A}(\varphi)$ the element, the ordered pair, or triple consisting of the corresponding elements in $\{c(\varphi), i(\varphi), n(\varphi), c(\varphi)+n(\varphi)\}$. For a $C^{\infty}$ map $\varphi_{0}: M \rightarrow N$, we say that a stable map $\varphi: M \rightarrow N$ has an A-minimal contour for $\varphi_{0}$ if $\mathbb{A}(\varphi)$ is minimal with respect to the lexicographic order among those stable maps which are homotopic to $\varphi_{0}$.

Let $\mathbb{A}=(i, c, n)$. The $(i, c, n)$-minimal contours were studied in [Demoto 2005; Kamenosono and Yamamoto 2009; Pignoni 1993]. The $(i, c, n)$-minimal contours of a $C^{\infty}$ map $\Sigma_{g} \rightarrow S^{2}$ of degree $d$ correspond to the bottom left corner of the lattice $L_{1, g+d} \cap D$. Note that for a $C^{\infty}$ map $M \rightarrow N$, there is a stable map with $S(f)$ consisting of one component.

This paper is organized as follows. In Section 2, we prepare some stable maps $M \rightarrow \mathbb{R}^{2}$ and $M \rightarrow S^{2}$ which we employ in the following section. In Section 3, we prove Theorems 1.1 and 1.2. In Section 4, we pose two problems which concern the apparent contours of stable maps between surfaces. In the Appendix, we study $i$ - $(c, n)$-minimal contours and $i$ - $(n, c)$-minimal contours of stable maps $\Sigma_{g} \rightarrow S^{2}$.

\section{Stable maps $\Sigma_{g} \rightarrow N\left(\right.$ for $N=\mathbb{R}^{2}$ or $\left.N=S^{2}\right)$}

In this section, we show that there exist stable maps $\Sigma_{g} \rightarrow \mathbb{R}^{2}$ and $\Sigma_{g} \rightarrow S^{2}$ whose triples $(i, c, n)$ are in the lists of Theorems 1.1 and 1.2, respectively. For two integers $k$ and $\ell$, set $\delta_{k, \ell}=2$ if $k \equiv \ell$, and $\delta_{k, \ell}=0$ otherwise. 
2A. Stable maps $\boldsymbol{\Sigma}_{\boldsymbol{g}} \rightarrow \mathbb{R}^{\mathbf{2}}$. Let $p_{S^{2}}: S^{2} \rightarrow \mathbb{R}^{2}$ be the standard projection defined by $(x, y, z) \mapsto(x, y)$. Then, the contour $\gamma\left(p_{S^{2}}\right)$ is an embedded circle in $\mathbb{R}^{2}$, namely, the triple $(i, c, n)$ is equal to $(1,0,0)$. Then, by applying modifications (1) and (2) to $p_{S^{2}}$ inductively, for each $(c, n)$ in

$$
L_{1,0} \cap\left\{(x, y) \mid x \geq 0, y \geq \frac{1}{2} x\right\},
$$

we obtain a stable map $f: S^{2} \rightarrow \mathbb{R}^{2}$ with $S(f)$ consisting of one component, $c$ cusps, and $n$ nodes. Furthermore, for a given integer $i \geq 1$, by applying modifications (7) and (8) $i-1$ times to stable maps $S^{2} \rightarrow \mathbb{R}^{2}$ whose pairs $(c, n)$ are in

$$
L_{1,0} \cap\left\{(x, y) \mid x \geq 0, y \geq \frac{1}{2} x\right\},
$$

for each $(c, n)$ in

$$
L_{i, 0} \cap\left\{(x, y) \mid x \geq \delta_{i, 0}, y \geq 0, y \geq \frac{1}{2} x-i+1\right\},
$$

we obtain a stable map $f: S^{2} \rightarrow \mathbb{R}^{2}$ with $S(f)$ consisting of $i$ components, $c$ cusps, and $n$ nodes.

Then, for given integers $g \geq 1$ and $i \geq 1$, with $i>g$, let $k$ and $\ell$ be nonnegative integers satisfying $k+\ell=g$. By applying modifications (4) $k$ times and (5) $\ell$ times to stable maps $S^{2} \rightarrow \mathbb{R}^{2}$ whose pairs $(c, n)$ are in

$$
L_{i, 0} \cap\left\{(x, y) \mid x \geq \delta_{i, 0}, y \geq 0, y \geq \frac{1}{2} x-i+1\right\},
$$

for each $(c, n)$ in

$$
L_{i, g} \cap\left\{(x, y) \mid x \geq \delta_{i, g}, y \geq 0, y \geq \frac{1}{2} x-g-i+1\right\},
$$

we obtain a stable map $f: \Sigma_{g} \rightarrow \mathbb{R}^{2}$ with $S(f)$ consisting of $i$ components, $c$ cusps, and $n$ nodes.

Thus, we obtain stable maps $\Sigma_{g} \rightarrow \mathbb{R}^{2}$ whose pairs $(c, n)$ are in the list of Theorem 1.1 with $i>g$.

Proposition 2.1. Let $g \geq 1$. For each pair $(c, n)$ in

$$
L_{1, g} \cap\left\{(x, y) \mid y=-\frac{1}{2} x+g+2, y \geq 1\right\},
$$

there is a stable map $\Sigma_{g} \rightarrow \mathbb{R}^{2}$ with $S(f)$ consisting of one component, c cusps, and $n$ nodes.

Proof. There exist stable maps $T^{2} \rightarrow \mathbb{R}^{2}$ whose triples $(i, c, n)$ are equal to $(1,2,2)$ and $(1,4,1)$. There also exist stable maps $\Sigma_{2} \rightarrow \mathbb{R}^{2}$ whose triples $(i, c, n)$ are equal to $(1,0,4)$ and $(1,2,3)$. See [Pignoni 1993; Yamamoto 2010] for the details.

By applying modifications (5) and (6) to the above four stable maps $T^{2} \rightarrow \mathbb{R}^{2}$, $\Sigma_{2} \rightarrow \mathbb{R}^{2}$ inductively, we obtain the desired stable maps $\Sigma_{g} \rightarrow \mathbb{R}^{2}$. For example, let us consider the case $g=2$. By applying modification (5) to stable maps $T^{2} \rightarrow \mathbb{R}^{2}$ 
whose triples $(i, c, n)$ are equal to $(1,2,2)$ and $(1,4,1)$, we obtain stable maps $\Sigma_{2} \rightarrow \mathbb{R}^{2}$ whose triples $(i, c, n)$ are equal to $(1,4,2)$ and $(1,6,1)$, respectively. Furthermore, let us consider the case $g=3$. By applying modification (6) to stable maps $T^{2} \rightarrow \mathbb{R}^{2}$ whose triples $(i, c, n)$ are equal to $(1,2,2)$ and $(1,4,1)$, we obtain stable maps $\Sigma_{3} \rightarrow \mathbb{R}^{2}$ whose triples $(i, c, n)$ are equal to $(1,2,4)$ and $(1,4,3)$, respectively. By applying modification (5) to stable maps $\Sigma_{2} \rightarrow \mathbb{R}^{2}$ whose triples $(i, c, n)$ are equal to $(1,4,2)$ and $(1,6,1)$, we obtain stable maps $\Sigma_{3} \rightarrow \mathbb{R}^{2}$ whose triples $(i, c, n)$ are equal to $(1,6,2)$ and $(1,8,1)$, respectively.

Then, by applying modifications (1) and (2) inductively to stable maps in Proposition 2.1, for each $(c, n)$ in

$$
L_{1, g} \cap\left\{(c, n) \mid x \geq \delta_{1, g}, y \geq 1, y \geq-\frac{1}{2} x+g+2, y \geq \frac{1}{2} x-g-2\right\},
$$

we obtain a stable map $\Sigma_{g} \rightarrow \mathbb{R}^{2}$ with $S(f)$ consisting of one component, $c$ cusps, and $n$ nodes.

For given integers $g \geq 1$ and $i \geq 1$ with $1 \leq i \leq g$, by applying modification (4) $i-1$ times to stable maps $\Sigma_{g-i+1} \rightarrow \mathbb{R}^{2}$ whose pairs $(c, n)$ are in

$$
\begin{aligned}
& L_{1, g-i+1} \cap\left\{(c, n) \mid x \geq \delta_{1, g-i+1}, y \geq 1, y \geq-\frac{1}{2} x+(g-i+1)+2,\right. \\
& y\left.\geq \frac{1}{2} x-(g-i+1)-2\right\},
\end{aligned}
$$

for each $(c, n)$ in

$$
L_{i, g} \cap\left\{(c, n) \mid x \geq \delta_{i, g}, y \geq 1, y \geq-\frac{1}{2} x+g+2, y \geq \frac{1}{2} x-g-2\right\},
$$

we obtain a stable map $\Sigma_{g} \rightarrow \mathbb{R}^{2}$ with $S(f)$ consisting of $i$ components, $c$ cusps, and $n$ nodes.

Thus, we obtain all stable maps $\Sigma_{g} \rightarrow \mathbb{R}^{2}$ whose triples $(i, c, n)$ are in the list of Theorem 1.1.

2B. Stable maps $\Sigma_{g} \rightarrow S^{\mathbf{2}}$. Note that stable maps $\Sigma_{g} \rightarrow \mathbb{R}^{2}$ obtained in Section 2A induce degree zero stable maps $\Sigma_{g} \rightarrow S^{2}$.

Let us consider stable maps $S^{2} \rightarrow S^{2}$. Denote by $f_{(1,2,0)}^{S^{2}, S^{2}}$ a degree one stable map $S^{2} \rightarrow S^{2}$ whose contour is shown in Figure 12 .

Let $d \geq 1$ and $i \geq 1$ be integers with $i \leq d$. By applying modifications (1), (2), and (3) inductively to a degree $d$ stable map $S^{2} \rightarrow S^{2}$ whose triple $(i, c, n)$ is equal to $(1,2 d, 0)$, for each $(c, n)$ in

$$
L_{1, d} \cap\{(x, y) \mid x \geq 2 d, y \geq 0\},
$$

we obtain a degree $d$ stable map $f: S^{2} \rightarrow S^{2}$ with $S(f)$ consisting of one component, $c$ cusps, and $n$ nodes. Then, by applying modification (10) $i-1$ times inductively 


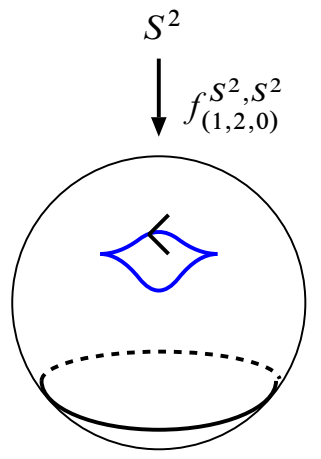

Figure 12. Stable map $f_{(1,2,0)}^{S^{2}, S^{2}}: S^{2} \rightarrow S^{2}$.

to these degree $d-i+1$ stable maps $S^{2} \rightarrow S^{2}$ whose pairs $(c, n)$ are in

$$
L_{1, d-i+1} \cap\{(x, y) \mid x \geq 2(d+1-i), y \geq 0\},
$$

for each $(c, n)$ in

$$
L_{i, d} \cap\{(x, y) \mid x \geq 2(d+1-i), y \geq 0\},
$$

we obtain a degree $d$ stable map $f: S^{2} \rightarrow S^{2}$ with $S(f)$ consisting of $i$ components, $c$ cusps, and $n$ nodes.

Let $d \geq 0$ and $i \geq 1$ be integers with $i \geq d$. By applying modification (10) $d$ times and $d-1$ times inductively to degree zero stable maps $S^{2} \rightarrow S^{2}$ and degree one stable maps $S^{2} \rightarrow S^{2}$ whose pairs $(c, n)$ are in

$$
L_{i, 0} \cap\left\{(x, y) \mid x \geq \delta_{i, 0}, y \geq 0\right\} \quad \text { and } \quad L_{i, 1} \cap\left\{(x, y) \mid x \geq \delta_{i, 1}, y \geq 0\right\}
$$

respectively, for each $(c, n)$ in

$$
L_{i, d} \cap\left\{(x, y) \mid x \geq \delta_{i, d}, y \geq 0\right\},
$$

we obtain a degree $d$ stable map $S^{2} \rightarrow S^{2}$ with $S(f)$ consisting of $i$ components, $c$ cusps and $n$ nodes.

Thus, we obtain all stable maps $S^{2} \rightarrow S^{2}$ whose pairs $(c, n)$ are in the lists of Theorem 1.2 with $g=0$.

In the following, assume $g \geq 1$. Let us consider degree zero stable maps $\Sigma_{g} \rightarrow S^{2}$ which are not induced from stable maps $\Sigma_{g} \rightarrow \mathbb{R}^{2}$.

Proposition 2.2. Let $g \geq 1$ and $i \geq 1$ with $i \leq g$. For each pair $(c, n)$ in

$$
L_{i, g} \cap\left\{(x, y) \mid x \geq \delta_{i, g}, y \geq 0, y=-\frac{1}{2} x+g+3-i\right\},
$$

there is a degree zero stable map $\Sigma_{g} \rightarrow S^{2}$ with $S(f)$ consisting of $i$ components, c cusps, and n nodes. 
Proof. For each $(c, n)$ in

$$
L_{i, g} \cap\left\{(x, y) \mid x \geq \delta_{i, g}, y \geq 1, y=-\frac{1}{2} x+g+3-i\right\},
$$

we already obtained a degree zero stable map $f: \Sigma_{g} \rightarrow S^{2}$ with $S(f)$ consisting of $i$ components, $c$ cusps and $n$ nodes in Section 2A.

By attaching a sphere which is mapped by orientation reversely to $f_{(1,2,0)}^{S^{2}, S^{2}}$, we obtain a degree zero stable map $S^{2} \rightarrow S^{2}$ whose triple $(i, c, n)$ is equal to $(1,4,0)$. Then, for each integers $g \geq 1$ and $i \geq 1$ with $i \leq g$, by applying modifications (4) $i-1$ times and (5) $g-i+1$ times to this degree zero stable map $S^{2} \rightarrow S^{2}$, we obtain a degree zero stable map $f: \Sigma_{g} \rightarrow S^{2}$ with $S(f)$ consisting of $i$ components, $2(g+3-i)$ cusps, and no nodes.

Let $g \geq 1$ and $i \geq 1$ with $i \leq g$. By applying modifications (1), (2), and (3) to stable maps obtained in the above subsection and in Proposition 2.2, for each pair $(c, n)$ in

$$
L_{i, g} \cap\left\{(x, y) \mid x \geq \delta_{i, g}, y \geq 0, y \geq-\frac{1}{2} x+g+3-i\right\},
$$

we obtain a stable map $\Sigma_{g} \rightarrow S^{2}$ with $S(f)$ consisting of $i$ components, $c$ cusps, and $n$ nodes.

Let $g \geq 1$ and $i \geq 1$ with $i>g$. By applying modifications (1), (2), and (3) inductively to a degree zero stable map $\Sigma_{g} \rightarrow S^{2}$ whose triple $(i, c, n)$ is equal to $\left(i, \delta_{i, g}, 0\right)$, for each $(c, n)$ in

$$
L_{i, g} \cap\left\{(x, y) \mid x \geq \delta_{i, g}, y \geq 0\right\},
$$

we obtain a degree zero stable map $f: \Sigma_{g} \rightarrow S^{2}$ with $S(f)$ consisting of $i$ components, $c$ cusps and $n$ nodes.

Thus, we obtain degree zero stable maps $\Sigma_{g} \rightarrow S^{2}$ in the lists of Theorem 1.2 (1) and (2).

In the following, assume $g \geq 1$ and $d \geq 1$.

Proposition 2.3. (1) If $g \leq d$, then for each $(c, n)$ in

$$
L_{1, g+d} \cap\left\{(x, y) \mid x \geq 2(d-g), y=-\frac{1}{2} x+g+d+2\right\},
$$

there is a degree d stable map $\Sigma_{g} \rightarrow S^{2}$ with $S(f)$ consisting one component, c cusps, and n nodes.

(2) If $d \leq g$, then for each $(c, n)$ in

$$
L_{1, g+d} \cap\left\{(x, y) \mid y=-\frac{1}{2} x+g+d+2, y \geq 3\right\},
$$

there is a degree d stable map $\Sigma_{g} \rightarrow S^{2}$ with $S(f)$ consisting one component, c cusps, and $n$ nodes. 
Proof. Stable maps $\Sigma_{g} \rightarrow \mathbb{R}^{2}$ and $\Sigma_{g} \rightarrow S^{2}$ whose triples $(i, c, n)$ are one of the following lists (1) and (2) were obtained in [Kamenosono and Yamamoto 2009; Yamamoto 2010] respectively:

(1) Stable maps $f: \Sigma_{g} \rightarrow \mathbb{R}^{2}$ whose triples $(i, c, n)$ are

$$
(i, c, n)= \begin{cases}(1,0,0) & \text { if } g=0 \\ (1,2, g+1) & \text { if } g \text { is odd } \\ (1,0, g+2) & \text { otherwise }\end{cases}
$$

Degree $d \geq 0$ stable maps $f: \Sigma_{g} \rightarrow S^{2}$ whose triples $(i, c, n)$ are

$$
(i, c, n)= \begin{cases}(1,2 d, 0) & \text { if } g=0, \\ (1,2(d-g), 2 g+2) & \text { if } d \geq g \geq 1, \\ (1,0, d+g+2) & \text { if } d \leq g \text { and } d \equiv g, \\ (1,2, d+g+1) & \text { if } d<g \text { and } d \neq \equiv .\end{cases}
$$

(2) Stable maps $f: \Sigma_{g} \rightarrow \mathbb{R}^{2}$ whose triples $(i, c, n)$ are

$$
(i, c, n)= \begin{cases}(1,0,0) & \text { if } g=0, \\ (1,2 g+2,1) & \text { otherwise }\end{cases}
$$

Degree $d \geq 0$ stable maps $f: \Sigma_{g} \rightarrow S^{2}$ whose triples $(i, c, n)$ are

$$
(i, c, n)= \begin{cases}(1,2 d, 0) & \text { if } g=0, \\ (1,2(g+2), 0) & \text { if } d=0 \text { and } g \geq 1, \\ (1,2(d+g), 0) & \text { otherwise. }\end{cases}
$$

On the other hand, there exists a degree one stable map $T^{2} \rightarrow S^{2}$ whose triple $(i, c, n)$ is equal to $(1,2,3)$; see [Kamenosono and Yamamoto 2009] for the details.

By applying modification (5) or (6), (9), (11) for stable maps $\Sigma_{g} \rightarrow \mathbb{R}^{2}$ and $\Sigma_{g} \rightarrow S^{2}$ in these lists, and a stable map $T^{2} \rightarrow S^{2}$ whose triple $(i, c, n)$ is equal to $(1,2,3)$, we obtain the desired stable maps $\Sigma_{g} \rightarrow S^{2}$.

Then, by applying modifications (1) and (2), (3), (10) inductively to stable maps $\Sigma_{g} \rightarrow S^{2}$ in Proposition 2.3, we obtain each stable map $\Sigma_{g} \rightarrow S^{2}$ in the list of Theorem 1.2(3) and (4).

Let $i \geq 1$ with $i \geq g+d$. By applying modifications (1) and (2), (3), (10) to degree zero stable maps $\Sigma_{g} \rightarrow S^{2}$ whose triples $(i, c, n)$ are $(g+1,0,0)$ and $(g+2,2,0)$, we obtain each stable map $\Sigma_{g} \rightarrow S^{2}$ in the list of Theorem 1.2(5).

Thus, we obtain all stable maps $\Sigma_{g} \rightarrow S^{2}$ whose triples $(i, c, n)$ are in the lists of Theorem 1.2. 


\section{Proof of Theorems 1.1 and 1.2}

3A. Preparation. In this subsection, some notions concerning the apparent contour of a stable map $M \rightarrow S^{2}$ of a closed surface are introduced, where $M$ is a closed surface and $S^{2}$ is oriented.

Let $\varphi: M \rightarrow S^{2}$ be a stable map whose contour is nonempty. Let $S(\varphi)=$ $S_{1} \cup \cdots \cup S_{\ell}$ be the decomposition of $S(\varphi)$ into the connected components and set $\gamma_{i}=\varphi\left(S_{i}\right)(i=1, \ldots, \ell)$. Note that $\gamma(\varphi)=\gamma_{1} \cup \cdots \cup \gamma_{\ell}$. Let $m(\varphi)$ be the smallest number of elements in the set $\varphi^{-1}(y)$, where $y \in S^{2}$ runs over all regular values of $\varphi$. Fix a regular value $\infty$ such that $\varphi^{-1}(\infty)$ consists of $m(\varphi)$ points. For each $\gamma_{i}$, denote by $U_{i}$ the component of $S^{2} \backslash \gamma_{i}$ which contains $\infty$. Note that $\partial U_{i} \subset \gamma_{i}$.

Orient $\gamma_{i}$ so that at each fold point image, the surface is "folded to the left hand side." More precisely, for a point $y \in \gamma_{i}$ which is not a cusp or a node, choose a normal vector $v$ of $\gamma_{i}$ at $y$ such that $\varphi^{-1}\left(y^{\prime}\right)$ contains more elements than $\varphi^{-1}(y)$, where $y^{\prime}$ is a regular value of $\varphi$ close to $y$ in the direction of $v$. Let $\tau$ be a tangent vector of $\gamma_{i}$ at $y$ such that the ordered pair $(\tau, v)$ is compatible with the given orientation of $S^{2}$. It is easy to see that $\tau$ gives a well-defined orientation for $\gamma_{i}$.

Definition 3.1. A point $y \in \partial U_{i} \backslash\{$ cusps, nodes $\}$ is said to be positive if the normal orientation $v$ at $y$ points toward $U_{i}$. Otherwise, it is said to be negative.

A component $\gamma_{i}$ is said to be positive if all points of $\partial U_{i} \backslash$ \{cusps, nodes\} are positive; otherwise, $\gamma_{i}$ is said to be negative. The number of positive and negative components is denoted by $i^{+}$and $i^{-}$, respectively. Note that there is at least one negative component unless $S(f)=\varnothing$.

Definition 3.2. A point $y \in \partial U_{i} \backslash$ \{cusps, nodes\} is called an admissible starting point if $y$ is a positive point of a positive component $\gamma_{i}$ (or a negative point of a negative component). Note that for each $i$, there always exists an admissible starting point on $\gamma_{i}$.

Definition 3.3. Suppose that $y \in \gamma_{i}$ is an admissible starting point and $Q \in \gamma_{i}$ is a node. Let $\alpha:[0,1] \rightarrow \gamma_{i}$ be a parametrization consistent with the orientation, singular only when the image is a cusp such that $\alpha^{-1}(y)=\{0,1\}$. Then, there are two numbers $0<t_{1}<t_{2}<1$ satisfying $\alpha\left(t_{1}\right)=\alpha\left(t_{2}\right)=Q$.

We say that $Q$ is positive if the orientation of $S^{2}$ at $Q$ defined by the ordered pair $\left(\alpha^{\prime}\left(t_{1}\right), \alpha^{\prime}\left(t_{2}\right)\right)$ coincides with that of $S^{2}$ at $Q$; negative, otherwise.

The number of positive nodes on $\gamma_{i}$ is denoted by $N_{i}^{+}$(and negative nodes by $N_{i}^{-}$). The definition of a positive or negative node on $\gamma_{i}$ depends on the choice of an admissible starting point $y$. However, it is known that the difference $N_{i}^{+}-N_{i}^{-}$ does not depend on the choice of $y$; see [Whitney 1941] for details. Thus, the number $N^{+}-N^{-}=\sum_{i=1}^{k}\left(N_{i}^{+}-N_{i}^{-}\right)$is well defined. Note that nodes arising from $\gamma_{i} \cap \gamma_{j}(i \neq j)$ play no role in the computation. 
Then, we obtain the following as an easy application of Pignoni's formula. Proposition 3.4 [Kamenosono and Yamamoto 2009; Pignoni 1993]. For a stable map $\varphi: M \rightarrow S^{2}$ of a closed surface of genus $g$, we have

$$
g=\varepsilon(M)\left(\left(N^{+}-N^{-}\right)+\frac{1}{2} c(\varphi)+\left(1+i^{+}-i^{-}\right)-m(\varphi)\right),
$$

where $\varepsilon(M)$ is equal to 1 if $M$ is orientable, and 2 otherwise.

Note that even if a 5-tuple $\left(N^{+}, N^{-}, c, i^{+}, i^{-}\right)$satisfies formula (3-1), there may not be a stable map $f: M \rightarrow S^{2}$ with $S(f)$ consisting of $i^{+}+i^{-}$components, $c$ cusps, and $N^{+}+N^{-}$nodes.

In the following of this section, we assume that $\gamma_{i} \cap \gamma_{j}=\varnothing$ if $i \neq j$ because we study the minimal number of nodes. Denote by $U_{\infty} \subset S^{2} \backslash \gamma(\varphi)$ the component which contains $\infty$. Denote by $\gamma_{1}$ the component of $\gamma(\varphi)$ which contains $\partial U_{\infty}$. Note that $\gamma_{1}$ is a negative component of $\varphi$. Then, the following lemmas and corollary were obtained by Fukuda and Yamamoto.

Lemma 3.5 [Yamamoto 2010]. If $\gamma_{1}$ has a node, then it has a negative node.

Lemma 3.6 [Yamamoto 2010]. If a positive component $\gamma_{i}$ has a node, then it has a positive node.

Corollary 3.7 [Fukuda and Yamamoto 2011]. If the number of negative components of $\gamma(\varphi)$ is equal to one and $\gamma(\varphi)$ has a node, then it has a negative node.

Corollary 3.7 implies the following corollary.

Corollary 3.8. If the number of negative components of $\gamma(\varphi)$ is equal to one and $\gamma_{1}$ has no node, then it has no node.

Formula (3-1) and Lemma 3.6 imply the following lemma.

Lemma 3.9. Suppose that $g \geq 1$ and $f: \Sigma_{g} \rightarrow \mathbb{R}^{2}$ is a stable map with $2 \leq i(f) \leq g$. If $\gamma_{1}$ has no node, then $\gamma(f)$ has at least two negative components.

Proof. Assume that $\gamma(f)$ has only one negative component. Then, Lemma 3.6 and the assumption imply that $\gamma(f)$ has no node. Then, by the geometrical condition for a cusp, $\gamma(f)$ has no cusps. Thus, the formula (3-1) implies the contradiction

$$
0 \leq g-i(f)=-1 .
$$

By formula (3-1) and the three modifications (1), (2), and (3), in order to prove Theorem 1.1, for a given triple $(g, i, c)$, we only have to study the minimal number of nodes among stable maps $f: \Sigma_{g} \rightarrow \mathbb{R}^{2}$ with $S(f)$ consisting of $i$ components and $c$ cusps. Analogously, in order to prove Theorem 1.2, for a given 4-tuple $(g, d, i, c)$, we only have to study the minimal number of nodes among degree $d$ stable maps $f: \Sigma_{g} \rightarrow S^{2}$ with $S(f)$ consisting of $i$ components and $c$ cusps. 
Note that for a fixed pair $\left(i^{+}, i^{-}\right)$, if we increase the number of negative node by one, there are two ways to satisfy (3-1). One way is to increase the number of cusps by two. This corresponds to modification (1). The other way is to increase the number of positive node by one. This corresponds to modification (2).

Lemma 3.10. Let $g \geq 0$ and $d \geq 0$. If a degree $d$ stable map $f: \Sigma_{g} \rightarrow S^{2}$ satisfies

$$
\frac{1}{2} c(f) \equiv g+d+i(f) \bmod 2,
$$

then $f$ has at least one node.

Proof. If $f$ has no node, then (3-1) implies that

$$
g+m(f)+2 i^{-}=\frac{1}{2} c(f)+1+i(f) .
$$

In particular, Lemma 3.10 implies that if a stable map $f: \Sigma_{g} \rightarrow \mathbb{R}^{2}$ satisfies $\frac{1}{2} c(f) \equiv g+i(f) \bmod 2$, then $f$ has at least one node.

We recall a formula obtained by Eliasberg and Quine.

Theorem 3.11 [Eliashberg 1970; Quine 1978]. For a stable map $f: M \rightarrow N$ between closed connected oriented surfaces, we have

$$
\chi(M)-2 \chi\left(M_{-}\right)+\sum_{q_{k}} \operatorname{sig} \text { a cusp } \operatorname{sign}\left(q_{k}\right)=(\operatorname{deg} f) \chi(N)
$$

where $\chi$ denotes the Euler characteristic, $\operatorname{deg} f$ denotes the mapping degree of $f$, $M_{-}$is the closure of the set of regular points whose neighborhoods are mapped by $f$ in an orientation reversing way, and $\operatorname{sign}\left(q_{k}\right)= \pm 1$ is the sign of a cusp $q_{k}$ defined as the local mapping degree.

Then, Theorem 3.11 implies the following lemma.

Lemma 3.12. Let $g \geq 0, d \geq 0$, and $f: \Sigma_{g} \rightarrow S^{2}$ be a degree $d$ stable maps. Then, $\gamma(f)$ has at least $2(d+1-g-i)$ cusps.

Proof. Formula (3-2) implies that

$$
\sum_{q_{k} \text { is a cusp }} \operatorname{sign}\left(q_{k}\right)=2\left(d+g-1+\chi\left(\left(\Sigma_{g}\right)_{-}\right) .\right.
$$

On the other hand, we have $\chi\left(\Sigma_{g}\right)-i \leq \chi\left(\left(\Sigma_{g}\right)_{-}\right) \leq i$.

In particular, for a stable map $f: \Sigma_{g} \rightarrow \mathbb{R}^{2}, \gamma(f)$ has at least $2(g+1-i)$ cusps. Theorem 3.11 also implies the following lemma.

Lemma 3.13 [Fukuda and Yamamoto 2011]. Let $d \geq 0$ and $f: \Sigma_{g} \rightarrow S^{2}$ be a degree $d$ stable map. If $i(f) \equiv d+g \bmod 2$, then $\gamma(f)$ has at least two cusps.

In particular, for a stable map $f: M \rightarrow \mathbb{R}^{2}$, if $i(f) \equiv g \bmod 2$, then $\gamma(f)$ has at least two cusps.

Furthermore, if the contour has no nodes, then we have the following lemma. 
Lemma 3.14. (1) Let $g \geq 0$ and $f: \Sigma_{g} \rightarrow \mathbb{R}^{2}$ be a stable map with $2 \leq i(f) \leq g$. If $\gamma(f)$ has no nodes, then $c(f) \geq 2(g+3-i)$.

(2) Let $g \geq 0$ and $d \geq 0, f: \Sigma_{g} \rightarrow S^{2}$ be a degree d stable map with $2 \leq i(f) \leq g$. If $\gamma(f)$ has no nodes, then $c(f) \geq 2(g+d+1-i)$.

Proof. Let us consider (1). Then, formula (3-1) implies that

$$
g+2 i^{-}-1-i(f)=\frac{1}{2} c(f) .
$$

Then, Lemma 3.9 yields the conclusion.

The case (2) is also proved in a similar way.

\section{B. Proof of Theorem 1.1.}

Lemma 3.15. Let $g \geq 1$ and $f: \Sigma_{g} \rightarrow \mathbb{R}^{2}$ be a stable map with $1 \leq i(f) \leq g$. If $c(f) \leq 2(g+2-i(f))$, then $n(f) \geq-\frac{1}{2} c(f)+g+3-i(f)$.

Proof. Assume $i(f)=1$. In this case, for a stable map $f: \Sigma_{g} \rightarrow \mathbb{R}^{2}$ with $i(f)=1$, formula (3-1) implies that

$$
g-\frac{1}{2} c(f)=\left(N^{+}-N^{-}\right) .
$$

Then, Lemma 3.5 implies that

$$
n(f)=N^{+}+N^{-}=-\frac{1}{2} c(f)+g+2 N^{-} \geq-\frac{1}{2} c(f)+g+2 .
$$

Assume $i(f) \geq 2$. If the negative component $\gamma_{1}$ has a node, then formula (3-1) and Lemma 3.5 imply that

$$
n(f)=N^{+}+N^{-} \geq-\frac{1}{2} c(f)+g+3-i(f) .
$$

If the negative component $\gamma_{1}$ has no node, then formula (3-1) and Lemma 3.9 also imply that $n(f) \geq-\frac{1}{2} c(f)+g+3-i(f)$.

Lemma 3.16. Let $g \geq 0$ and $f: \Sigma_{g} \rightarrow \mathbb{R}^{2}$ be a stable map. If $c(f) \geq 2(g+i(f))$, then $N^{-} \geq \frac{1}{2} c(f)-g-i(f)+1$.

Proof. Formula (3-1) and the inequality $i^{+}-i^{-} \geq-i(f)$ imply that

$$
g \geq\left(N^{+}-N^{-}\right)+\frac{1}{2} c(f)+(1-i(f)) .
$$

Lemmas 3.10, 3.12, 3.13, 3.14(1), 3.15, and 3.16 prove Theorem 1.1 with $1 \leq i \leq g$. Lemmas 3.12, 3.13, 3.14(1) and 3.16 prove Theorem 1.1 with $i>g$.

This complete the proof of Theorem 1.1. 
3C. Proof of Theorem 1.2. Lemmas 3.12 and 3.13 prove Theorem 1.2 with $g=0$. Lemma 3.13 proves Theorem 1.2(2) and (5).

Lemma 3.17. Let $g \geq 1$. For a degree zero stable map $f: \Sigma_{g} \rightarrow S^{2}$, if $S(f)$ consists of one component and $\gamma(f)$ has no nodes, then $m(f) \geq 2$.

Proof. Under the assumption, formula (3-1) implies that

$$
g=\frac{1}{2} c(f)-m(f) .
$$

By the geometrical condition for a cusp, if $n(f)=0$ and $m(f)=0$, then $f$ has no cusps. Then, we have $g=0$, which is a contradiction.

Lemma 3.17 and formula (3-1) imply the following lemma.

Lemma 3.18. Let $g \geq 1$ and $f: \Sigma_{g} \rightarrow S^{2}$ be a degree zero stable map with $1 \leq i(f) \leq g$. If $c(f) \leq 2(g+3-i(f))$, then $n(f) \geq-\frac{1}{2} c(f)+g+3-i(f)$.

Proof. Formula (3-1) implies that

$$
n(f)=N^{+}+N^{-}=g+2 i^{-}-i(f)-\frac{1}{2} c(f)-1+2 N^{-} .
$$

Consider the case that $i(f)=1$. Then, by Lemma 3.5, $n(f) \geq-\frac{1}{2} c(f)+g+2$. Note that there is no degree zero stable map $f: \Sigma_{g} \rightarrow S^{2}$ with $S(f)$ consisting of one component and no nodes unless $g=0$.

Now consider the case that $2 \leq i(f) \leq g$. If $\gamma_{1}$ has a node, then Lemma 3.5 implies that

$$
n(f) \geq-\frac{1}{2} c(f)+g+3-i(f) .
$$

If $\gamma_{1}$ has no node, then Lemma 3.9 also implies the same inequality.

Let $f: \Sigma_{g} \rightarrow S^{2}$ be a degree zero stable map with no nodes and $2 \leq i(f) \leq g$. If $m(f)=0$, then $f$ induces a stable map $\Sigma_{g} \rightarrow \mathbb{R}^{2}$ whose triple $(i, c, n)$ is equal to that of $f$. Then, Lemma 3.9 and formula (3-1) imply that

$$
g+4 \leq g+2 i^{-}=\frac{1}{2} c(f)+(1+i(f)) .
$$

This inequality shows that $c(f) \geq 2(g+3-i(f))$.

If $m(f) \neq 0$, then Lemma 3.5 and formula (3-1) imply that

$$
g+4 \leq g+m(f)+2 i^{-}=\frac{1}{2} c(f)+(1+i(f)) .
$$

This inequality also shows that $c(f) \geq 2(g+3-i(f))$.

Thus, Lemma 3.10 and Lemmas 3.12, 3.18 prove Theorem 1.2(1).

Lemma 3.17 and formula (3-1) imply the following lemma.

Lemma 3.19. Let $g \geq 1$ and $d \geq 1, f: \Sigma_{g} \rightarrow S^{2}$ be a degree $d$ stable map with $1 \leq i(f) \leq g+d-1$. If $c(f) \leq 2(g+d-i)$, then $n(f) \geq-\frac{1}{2} c(f)+g+d+3-i(f)$. 
Proof. Let us consider the case $i(f)=1$. In this case, the formula (3-1) implies that

$$
n(f)=N^{+}+N^{-} \geq-\frac{1}{2} c(f)+g+d+2 N^{-}
$$

Then, Lemma 3.5 yields the conclusion.

Let us consider the case $2 \leq i(f) \leq g+d-1$. If $i^{-}=1$, then the formula (3-1) implies that

$$
n(f)=N^{+}+N^{-} \geq-\frac{1}{2} c(f)+g+d+1-i(f)+2 N^{-} .
$$

Thus, Lemma 3.5 and Corollary 3.8 yield the conclusion. If $i^{-} \geq 2$, then the formula (3-1) implies that

$$
n(f)=N^{+}+N^{-} \geq-\frac{1}{2} c(f)+g+d+3-i(f)+2 N^{-} .
$$

Thus, Lemma 3.10 and Lemmas 3.12, 3.19 prove Theorem 1.2(3) and (4). It completes the proof of Theorem 1.2.

\section{Problems}

In this section, we pose some problems which concern the number of the singularities of stable maps between surfaces.

Problem 4.1. Study the triples $(i, c, n)$ of stable maps $M \rightarrow N\left(N=\mathbb{R}^{2}\right.$ or $\left.N=S^{2}\right)$ of closed and nonorientable surfaces.

Pignoni [1993] (see also [Kamenosono and Yamamoto 2009]) observed that there are differences between $(i, c+n)$-minimal contours and $(i, c, n)$-minimal contours - see Remark 1.4 for the definitions - of $C^{\infty}$ maps of the real projective plane into $\mathbb{R}^{2}$ and $S^{2}$.

Figure 13 shows that the contours of stable maps $S^{2} \rightarrow \mathbb{R}^{2}$ whose triples $(i, c, n)$ are equal to $(2,2,2)$. Figure 14 also shows that the contours of stable maps $T^{2} \rightarrow \mathbb{R}^{2}$ whose triples $(i, c, n)$ are equal to $(2,0,4)$.

Problem 4.2. Introduce notions which distinguish two contours in Figure 13 (or 14). Then, study contours of stable maps between surfaces under the notions.
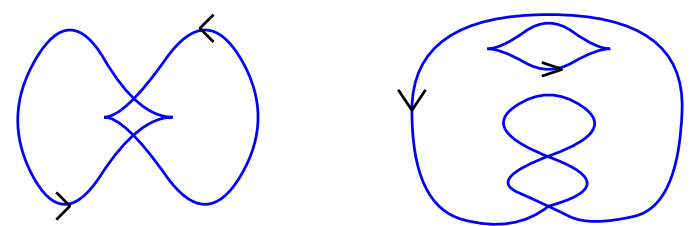

Figure 13. Contours of stable maps $S^{2} \rightarrow \mathbb{R}^{2}$ whose triples $(i, c, n)$ are $(2,2,2)$. 

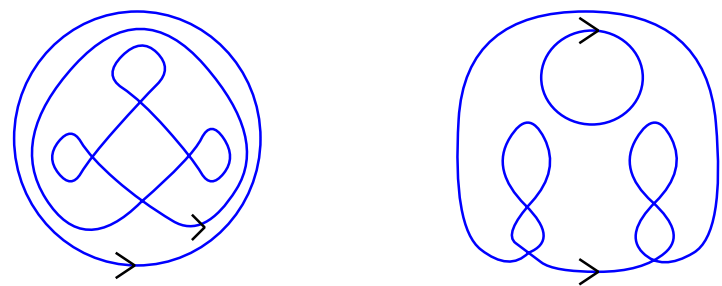

Figure 14. Contours of stable maps $T^{2} \rightarrow \mathbb{R}^{2}$ whose triples $(i, c, n)$ are $(2,0,4)$.

Problem 4.2 makes the second step toward classifying generic $C^{\infty}$ maps of closed surfaces into $\mathbb{R}^{2}$ or $S^{2}$ up to right-left equivalence.

\section{Appendix}

In this section, we introduce the notions of an $i-(c, n)$-minimal contour and an $i$ - $(n, c)$-minimal contour for a $C^{\infty}$ map $M \rightarrow N$ between surfaces. We study such minimal contours.

Taishi Fukuda and the author [Fukuda and Yamamoto 2011] studied $(c+n)$ minimal contours among stable maps $f: \Sigma_{g} \rightarrow S^{2}$ homotopic to a given $C^{\infty}$ map $\Sigma_{g} \rightarrow S^{2}$ such that $i(f)=i$, for each integer $i \geq 2$. Let us call such a minimal contour an $i-(c+n)$-minimal contour. Note that the case $g=2$ of [Fukuda and Yamamoto 2011, Theorem 1.2] has one error. The correct table of $i-(c+n)$-minimal contours for degree $d \geq 0$ stable maps $\Sigma_{2} \rightarrow S^{2}$ is the following:

$$
(c, n)= \begin{cases}(2(d-i-1), 6) & \text { if } 1 \leq i \leq d-1 \\ (2,4) \text { or }(6,0) & \text { if } i=d \\ (0,4) \text { or }(4,0) & \text { if } i=d+1 \\ (2,2) & \text { if }(d, i)=(0,2) \\ (2,0) & \text { if } i \geq d+2, i \equiv d \bmod 2, \text { except }(d, i)=(0,2) \\ (0,0) & \text { if } i \geq d+2, i \neq d \bmod 2\end{cases}
$$

For a nonnegative integer $i$, let us consider $(c, n)$-minimal contours among stable maps $f: \Sigma_{g} \rightarrow S^{2}$ homotopic to a given $C^{\infty}$ map $\Sigma_{g} \rightarrow S^{2}$ such that $i(f)=i$. Let us call such a minimal contour an $i-(c, n)$-minimal contour. Then, Theorems 1.1 and 1.2 imply the following proposition.

Proposition A.1. (1) The contour $\gamma(f)$ of a stable map $f: \Sigma_{g} \rightarrow \mathbb{R}^{2}$ is $i$ - $(c, n)$ minimal if and only if the pair $(c, n)$ is one of the following: 


$$
(c, n)= \begin{cases}(2, g+2-i) & \text { if } g \geq i \text { and } g \equiv i \bmod 2, \\ (0, g+3-i) & \text { if } g \geq i \text { and } g \neq i \bmod 2, \\ (2,0) & \text { if } g<i \text { and } g \equiv i \bmod 2, \\ (0,0) & \text { if } g<i \text { and } g \neq i \bmod 2 .\end{cases}
$$

(2) Let $f: \Sigma_{g} \rightarrow S^{2}$ be a degree $d \geq 0$ stable map such that $S(f)$ consists of $i$ components. Then, the contour $\gamma(f)$ is $i$ - $(c, n)$-minimal if and only if the pair $(c, n)$ for $\gamma(f)$ is one of the following:

$g=0$ :

$$
(c, n)= \begin{cases}(2(d-i+1), 0) & \text { if } 1 \leq i \leq d+1, \\ (2,0) & \text { if } i \geq d+2, i \equiv d \bmod 2, \\ (0,0) & \text { if } i \geq d+2, i \not \equiv d \bmod 2,\end{cases}
$$

$g \geq 1:$

$$
(c, n)= \begin{cases}(2(d-g-i+1), 2+2 g) & \text { if } 1 \leq i \leq d-g+1, \\ (2, d+g-i+2) & \text { if } d-g+2 \leq i<d+g-1, \\ & \text { and } i \equiv d+g \bmod 2, \\ (0, d+g-i+3) & \text { if } d-g+2 \leq i \leq d+g-1, \\ & \text { and } i \neq d+g \bmod 2, \\ (2,2) & \text { if }(d, i)=(0, g), \\ (2,0) & \text { if } i \geq d+g, i \equiv d+g \bmod 2, \\ & \text { except }(d, i)=(0, g), \\ (0,0) & \text { if } i \geq d+g, i \neq d+g \bmod 2 .\end{cases}
$$

Let us study $(n, c)$-minimal contours among stable maps $f: \Sigma_{g} \rightarrow S^{2}$ homotopic to a given $C^{\infty}$ map $\Sigma_{g} \rightarrow S^{2}$ such that $i(f)=i$, for each integer $i \geq 1$. Let us call such a minimal contour an $i$ - $(n, c)$-minimal contour. Then, Theorems 1.1 and 1.2 also imply the following proposition.

Proposition A.2. (1) The contour $\gamma(f)$ of a stable map $f: \Sigma_{g} \rightarrow \mathbb{R}^{2}$ is $i$ - $(n, c)$ minimal if and only if the pair $(c, n)$ is one of the following:

$i=1$ :

$$
(c, n)= \begin{cases}(0,0) & \text { if } g=0, \\ (2 g+2,1) & \text { otherwise }\end{cases}
$$

$i \geq 2$ :

$$
(c, n)= \begin{cases}(2(g+3-i), 0) & \text { if } g \geq i \\ (2,0) & \text { if } g<i \text { and } g \equiv i \bmod 2 \\ (0,0) & \text { otherwise }\end{cases}
$$


(2) Let $f: \Sigma_{g} \rightarrow S^{2}$ be a degree $d \geq 0$ stable map such that $S(f)$ consists of $i$ components. Then, the contour $\gamma(f)$ is $i-(n, c)$-minimal if and only if the pair $(c, n)$ for $\gamma(f)$ is one of the items below:

$$
(c, n)= \begin{cases}(2(g+3-i), 0) & \text { if } d=0 \text { and } 1 \leq i \leq g, \\ (2(d+g+1-i), 0) & \text { if } d \neq 0 \text { and } 1 \leq i \leq d+g-1, \\ (2,0) & \text { if } i \geq d+g \text { and } i \equiv d+g \bmod 2, \\ (0,0) & \text { if } i>d+g \text { and } i \neq d+g \bmod 2 .\end{cases}
$$

\section{Acknowledgments}

The author would like to express his gratitude to Andras Szúcs for the fruitful questions and comments at the International Workshop on Real and Complex Singularities held in São Carlos in 2012. He would like to thank Osamu Saeki for useful comments and suggestions which improved the paper. He would like to thank the referee for useful suggestions that helped him to improve the original manuscript. The author also expresses special thanks to Akiko Neriugawa for the useful advice in English grammar and for encouraging support. This work was supported by JSPS KAKENHI Grant Numbers 21740056 and 23654028.

\section{References}

[Demoto 2005] S.-I. Demoto, "Stable maps between 2-spheres with a connected fold curve", Hiroshima Math. J. 35:1 (2005), 93-113. MR 2006b:57041 Zbl 1080.57029

[Eliashberg 1970] Y. M. Eliashberg, “Об особенностях типа складки”, Izv. Akad. Nauk SSSR Ser. Mat. 34:5 (1970), 1110-1126. Translated as "On singularities of folding type" in Math. USSR Izv. 4:5 (1970), 1119-1134. MR 43 \#4051 Zbl 0226.57012

[Fukuda and Yamamoto 2011] T. Fukuda and T. Yamamoto, "Apparent contours of stable maps into the sphere", J. Singul. 3 (2011), 113-125. MR 2012k:58064 Zbl 1292.57022

[Kamenosono and Yamamoto 2009] A. Kamenosono and T. Yamamoto, "The minimal number of singularities of stable maps between surfaces", Topology Appl. 156:14 (2009), 2390-2405. MR 2010m:57041 Zbl 1207.57039

[Pignoni 1993] R. Pignoni, "Projections of surfaces with a connected fold curve", Topology Appl. 49:1 (1993), 55-74. MR 93m:57030 Zbl 0767.57020

[Quine 1978] J. R. Quine, "A global theorem for singularities of maps between oriented 2-manifolds", Trans. Amer. Math. Soc. 236 (1978), 307-314. MR 57 \#14020 Zbl 0379.57006

[Whitney 1941] H. Whitney, "On regular families of curves", Bull. Amer. Math. Soc. 47 (1941), 145-147. MR 2,322b Zbl 0025.23602

[Whitney 1955] H. Whitney, "On singularities of mappings of euclidean spaces, I: Mappings of the plane into the plane", Ann. of Math. (2) 62 (1955), 374-410. MR 17,518d Zbl 0068.37101

[Yamamoto 2009] M. Yamamoto, "The number of singular set components of fold maps between oriented surfaces”, Houston J. Math. 35:4 (2009), 1051-1069. MR 2011e:58064 Zbl 1186.57021 
[Yamamoto 2010] T. Yamamoto, "Apparent contours with minimal number of singularities", Kyushu J. Math. 64:1 (2010), 1-16. MR 2011m:57038 Zbl 1195.57064

Received August 9, 2014. Revised March 25, 2015.

TAKAHIRO YAMAMOTO

FACULTY OF ENGINEERING

KYUSHU SANGYO UNIVERSITY

3-1 Matsukadai 2-CHOME

HIGASHIKU

FUKUOKA 813-8503

JAPAN

yama.t@ip.kyusan-u.ac.jp 


\title{
PACIFIC JOURNAL OF MATHEMATICS
}

\author{
msp.org/pjm
}

Founded in 1951 by E. F. Beckenbach (1906-1982) and F. Wolf (1904-1989)

\section{EDITORS}

Don Blasius (Managing Editor)

Department of Mathematics

University of California

Los Angeles, CA 90095-1555

blasius@math.ucla.edu

\author{
Paul Balmer \\ Department of Mathematics \\ University of California \\ Los Angeles, CA 90095-1555 \\ balmer@math.ucla.edu \\ Robert Finn \\ Department of Mathematics \\ Stanford University \\ Stanford, CA 94305-2125 \\ finn@math.stanford.edu \\ Sorin Popa \\ Department of Mathematics \\ University of California \\ Los Angeles, CA 90095-1555 \\ popa@math.ucla.edu
}

\author{
Vyjayanthi Chari \\ Department of Mathematics \\ University of California \\ Riverside, CA 92521-0135 \\ chari@math.ucr.edu \\ Kefeng Liu \\ Department of Mathematics \\ University of California \\ Los Angeles, CA 90095-1555 \\ liu@math.ucla.edu \\ Jie Qing \\ Department of Mathematics \\ University of California \\ Santa Cruz, CA 95064 \\ qing@ cats.ucsc.edu
}

\section{PRODUCTION}

Silvio Levy, Scientific Editor, production@msp.org

\section{SUPPORTING INSTITUTIONS}

ACADEMIA SINICA, TAIPEI

CALIFORNIA INST. OF TECHNOLOGY

INST. DE MATEMÁTICA PURA E APLICADA

KEIO UNIVERSITY

MATH. SCIENCES RESEARCH INSTITUTE

NEW MEXICO STATE UNIV.

OREGON STATE UNIV.

\author{
STANFORD UNIVERSITY \\ UNIV. OF BRITISH COLUMBIA \\ UNIV. OF CALIFORNIA, BERKELEY \\ UNIV. OF CALIFORNIA, DAVIS \\ UNIV. OF CALIFORNIA, LOS ANGELES \\ UNIV. OF CALIFORNIA, RIVERSIDE \\ UNIV. OF CALIFORNIA, SAN DIEGO \\ UNIV. OF CALIF., SANTA BARBARA
}

\author{
Daryl Cooper \\ Department of Mathematics \\ University of California \\ Santa Barbara, CA 93106-3080 \\ cooper@math.ucsb.edu \\ Jiang-Hua Lu \\ Department of Mathematics \\ The University of Hong Kong \\ Pokfulam Rd., Hong Kong \\ jhlu@maths.hku.hk \\ Paul Yang \\ Department of Mathematics \\ Princeton University \\ Princeton NJ 08544-1000 \\ yang@math.princeton.edu
}

These supporting institutions contribute to the cost of publication of this Journal, but they are not owners or publishers and have no responsibility for its contents or policies.

See inside back cover or msp.org/pjm for submission instructions.

The subscription price for 2016 is US $\$ 440 /$ year for the electronic version, and $\$ 600 /$ year for print and electronic.

Subscriptions, requests for back issues and changes of subscribers address should be sent to Pacific Journal of Mathematics, P.O. Box 4163, Berkeley, CA 94704-0163, U.S.A. The Pacific Journal of Mathematics is indexed by Mathematical Reviews, Zentralblatt MATH, PASCAL CNRS Index, Referativnyi Zhurnal, Current Mathematical Publications and Web of Knowledge (Science Citation Index).

The Pacific Journal of Mathematics (ISSN 0030-8730) at the University of California, c/o Department of Mathematics, 798 Evans Hall \#3840, Berkeley, CA 94720-3840, is published twelve times a year. Periodical rate postage paid at Berkeley, CA 94704, and additional mailing offices. POSTMASTER: send address changes to Pacific Journal of Mathematics, P.O. Box 4163, Berkeley, CA 94704-0163.

PJM peer review and production are managed by EditFLOW ${ }^{\circledR}$ from Mathematical Sciences Publishers.

\section{PUBLISHED BY}

\section{mathematical sciences publishers \\ nonprofit scientific publishing}

http://msp.org/

(C) 2016 Mathematical Sciences Publishers 


\section{PACIFIC JOURNAL OF MATHEMATICS}

Volume $280 \quad$ No. $2 \quad$ February 2016

Topological Molino's theory

JESÚS A. ÁlVAREz LóPEZ and MANUEl F. MoreIRA GALICIA

Equivariant principal bundles and logarithmic connections on toric varieties

INDRANIL BISWAS, ARIJIT DEY and MAINAK PODDAR

On a spectral theorem in paraorthogonality theory

Kenier CASTILlo, RuYmán CRUZ-BARRoso and Francisco

PERDOMO-PÍO

Sigma theory and twisted conjugacy, II: Houghton groups and pure

symmetric automorphism groups

DACIBERG L. GONÇALVES and PARAMESWARAN SANKARAN

The second CR Yamabe invariant

PAK TUNG Ho

No hyperbolic pants for the 4-body problem with strong potential

CONNOR JACKMAN and RICHARD MONTGOMERY

Unions of Lebesgue spaces and $A_{1}$ majorants

Greg Knese, John E. M ${ }^{\mathrm{C}}$ CARThy and Kabe Moen

Complex hyperbolic $(3,3, n)$ triangle groups

JOHN R. PARKER, JiEYAN WANG and BAOHUA XIE

Topological aspects of holomorphic mappings of hyperquadrics from $\mathbb{C}^{2}$ to $\mathbb{C}^{3}$

\section{MICHAEL REITER}

2-Blocks with minimal nonabelian defect groups III

BENJAMIN SAMBALE

Number of singularities of stable maps on surfaces 